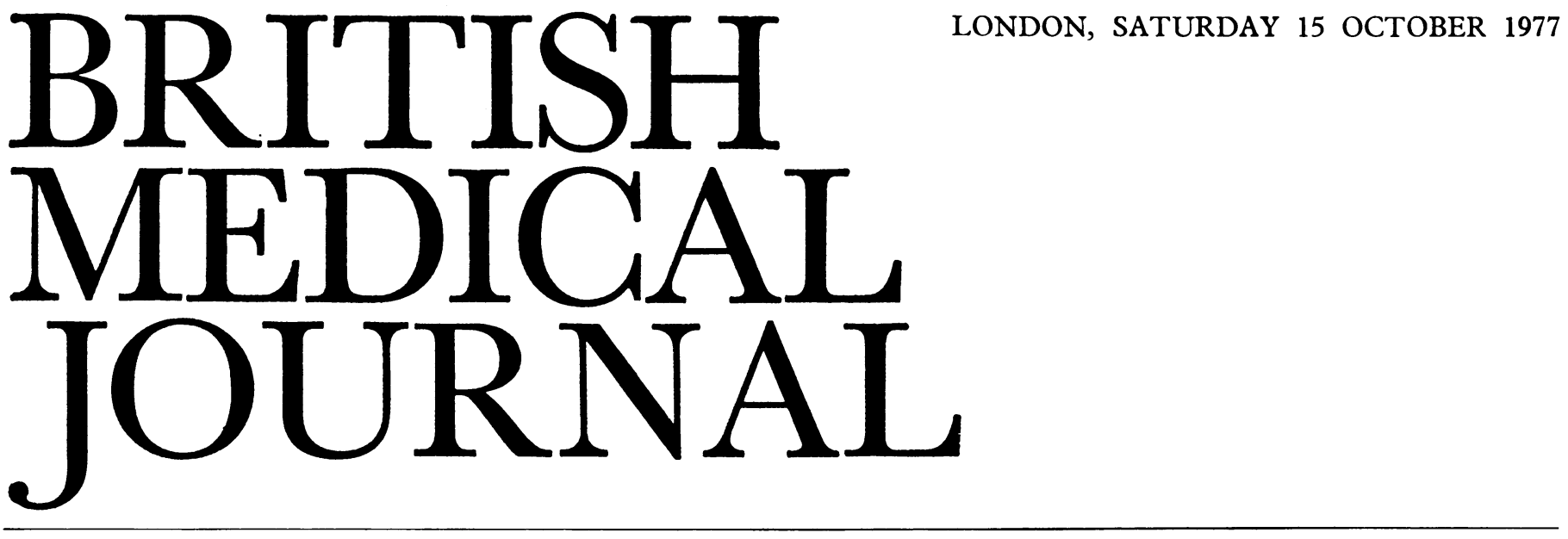

The great epidemics of scarlatina that swept through the crowded cities of Europe in the seventeenth to nineteenth centuries taught doctors that nephritis with bloody urine could follow the acute attack and on occasion kill the sufferer. ${ }^{1}$ When the streptococcus was isolated in 1881 it became the first specific antecedent of nephritis to be recognised. Previously, Bright had described the three classic phases of nephritis as one inexorable sequence: acute nephritis, nephrotic syndrome, uraemia, death. ${ }^{2}$ Immediately, others suggested that in fact these three archetypes might represent distinct diseases with the acute phase having a more benign outcome. Exponents of the unitary hypothesis explained the frequent absence of direct evidence connecting acute nephritis with the chronic phases by postulating a chronic latent phase of clinically quiescent or even inapparent disease after the acute episode followed by insidious entry into the later stages. This nineteenth-century debate is still with us.

Today in Britain most patients who present with an acute onset of haematuria accompanied by variable hypertension, oliguria, oedema, and uraemia - the acute nephritic syndrome - have no evidence of streptococcal infection as the precipitating factor. Indeed, acute poststreptococcal nephritis is now rare in Britain. ${ }^{3}$

The prognosis of children ${ }^{4-8}$ with poststreptococcal glomerulonephritis is better than that of adults ${ }^{9-11}$; in preschool children the disease is almost invariably benign. ${ }^{4}$ This gradient of prognosis with age is unexplained. A poorer prognosis is reflected in-and probably arises from-a higher incidence of histological abnormalities in the glomeruli normally associated with progressive disease. These abnormalities show several different patterns. Most often there is mainly endothelial and mesangial proliferation. A few patients have a combination of capillary wall lesions with deposits and low serum complement, which persist for eight weeks or more - the pattern of mesangiocapillary (membranoproliferative) glomerulonephritis. ${ }^{12}$ Another small group shows extensive proliferation of extracapillary cells to form crescents surrounding most glomeruli. ${ }^{13}$ Occasionally after streptococcal infection a patient changes from having a relatively benign mesangial, endocapillary nephritis to mesangiocapillary ${ }^{14}$ or crescentic glomerulonephritis. ${ }^{1516}$ It is difficult to know how many patients show these aberrant, more severe forms. Hospital series are biased by the selection of patients for biopsy in favour of an overestimate, but less than $5 \%$ of children with poststreptococcal nephritis follow either severe course, ${ }^{4-817}$ though perhaps more adults do so. ${ }^{9-11}$ Furthermore, most patients showing these appearances in a renal biopsy specimen, even in acute nephritis, have no evidence of antecedent streptococcal infection. ${ }^{12} 13$

These unfortunates represent only a minority of patients with poststreptococcal glomerulonephritis. But what is the prognosis of the majority, who show only endocapillary proliferation in their glomeruli, and whose early history usually suggests clinical and histological healing? What also is the prognosis of those patients who do not consult their doctor, but who (despite the absence of obvious disease) are found to have urinary abnormalities on testing or histological changes in the absence of even urinary abnormalities ? ${ }^{18}$ Do they account for many of the patients who develop renal failure without any evident history of glomerulonephritis?

The prognosis up to ten years later in children with both sporadic and epidemic poststreptococcal nephritis has appeared to be good, ${ }^{4-71718}$ provided, on initial or later biopsy, the patient has no mesangiocapillary or crescentic glomerulonephritis. Even among those with endocapillary disease, however, up to $25 \%$ show urinary abnormalities or hypertension, or both, in the decade after the attack, ${ }^{717}$ though renal function is almost invariably preserved. In adults the picture has been less reassuring, perhaps because of a higher incidence of mesangiocapillary and crescentic glomerulonephritis at onset, ${ }^{9-11}$ but those with only endocapillary glomerulonephritis have still seemed to have a good prognosis short term, ${ }^{9}$ although not as good as in children.

These relatively reassuring conclusions have been challenged over the past few years in reports from New York ${ }^{19-21}$ and Venezuela. ${ }^{22}$ The studies of Baldwin and his colleagues in New York ${ }^{19-21}$ have attracted considerable attention because of their conclusions that "more than half" of all patients with poststreptococcal glomerulonephritis followed for long periods show evidence of irreversible renal damage; that the absence of proteinuria or haematuria does not necessarily imply an inactive nephritis; and that sclerosis, far from representing the final stage of healing an acute process, indicates progressive renal destruction. Baldwin and his colleagues first reported on 126 patients in $1974^{19}$ and now on 168 in $1977 . .^{20}$ The data and conclusions remain essentially the same in these two reports except that a larger proportion of children are included in the second series.

One problem with this study (unlike that of the smaller but almost complete studies of Travis, ${ }^{5}$ Lewy, ${ }^{6}$ or Gill $^{7}$ ) is that 
only a proportion of patients have been traced or followed up: in the 1974 series $^{19}$ only 56 of the 126 patients were studied at one year, only 29 at five years or more, and only six at 10 years. In the second series the numbers were 68,33 , and 18 , respectively. More data were available 10 years after onset from even the small childhood series of Gill. ${ }^{7}$ This point is crucial, since almost all the data in Baldwin's papers are presented as percentages of the patients observed at a particular time-and the analysis makes the untestable (and in a relatively benign condition probably incorrect) assumption that most patients who were not traced were similar to those who remained in the study. Furthermore, the definition of "irreversible" was based on more assumptions: proteinuria does not necessarily indicate structural damage; the definition used for a reduced glomerular filtration rate was very strict; and only 35 "randomly selected" patients had their glomerular filtration rate determined.

Their most important suggestion, however, is that glomerulosclerosis represents a progressive disease, not healing. The evidence for this suggestion is more compelling, since the incidence of severe grades of sclerosis (complete glomerulosclerosis of over $10 \%$ of glomeruli) was found to increase as proliferation subsided, and these severe grades were present in $56^{\circ}$, of the 100 biopsy specimens obtained three or more years after the acute attack. Six patients, all adults, developed renal failure between three and 11 years later, ${ }^{21}$ despite the fact that their initial biopsy specimens had shown only endocapillary proliferation and that the plasma creatinine concentration had returned to normal after the acute attack in five of the six.

These data from Baldwin et al ${ }^{19} 20$ (possibly taken from patients with unusually severe disease) may differ less from those of other observers than at first appears. Most authors have concentrated attention on the majority of patients with normal function and apparent clinical healing; Baldwin emphasised the minority with continuing disease or progressive renal damage. The crucial issue is the size of this minority in patients who are found at first to have the classic benign pattern of endocapillary proliferation. As yet we have little evidence, even in Baldwin's data, that children in this category go into renal failure: no child in his 1974 series had a glomerular filtration rate of less than $80 \mathrm{ml}$ min. The New York group has, however, reminded us that patients may have hypertension and reduced renal function without urinary abnormalities and forced a reconsideration of the vexed questions of global and segmental glomerulosclerosis.

Physicians dealing with adults must worry about these conclusions more than paediatricians. Even so, it remains unlikely that most renal failure in Britain arises from undiagnosed streptococcal infection when the condition is so rare and yet so eagerly sought in patients with overt nephritis. Whether it plays a more important part in areas of the world where streptococcal infection and poststreptococcal nephritis are still common remains to be seen.

${ }^{1}$ Von Pleneitz, M A, Opera Medico-physica, Tract III. De Scarlatina, Vienna, Trattner 1762; Miller, J, Lancet, 1849, 2, 1, 57, 113, 124, 197, $281,524,685$.

2 Bright, R, Guy's Hospital Reports, 1836, 1, 388, 380.

3 Meadow, R, Archives of Disease in Childhood, 1976, 50, 379.

${ }^{4}$ Dodge, W F, et al, New England fournal of Medicine, 1972, 286, 273.

5 Travis, L B, et al, Clinical Nephrology, 1973, 1, 169.

${ }^{6}$ Lewy, J E, et al, Medicine (Baltimore), 1971, 50, 453

${ }^{7}$ Gill, D, et al, Archives of Disease in Childhood, 1977, 52, 423.

8 Perlman, L V, et al, fournal of the American Medical Association, 1965, 194, 63.

${ }^{9}$ Jennings, R B, and Earle, D P, fournal of Clinical Investigation, 1971, 40, 1525.

${ }^{10}$ McCluskey, R T, and Baldwin, D S, American fournal of Medicine, 1963, 35, 213.
${ }^{11}$ Kushner, D S, et al, Medicine (Baltimore), 1961, 40, 203.

${ }^{12}$ Habib, R, et al, Clinical Nephrology, 1973, 1, 194.

${ }^{13}$ Whitworth, J A, et al, Nephron, 1976, 16, 1.

14 Glasgow, E F, and White, R H R, in Glomerulonephritis, eds P KincaidSmith, T H Mathew, and E L Becker, vol 1, p 345. New York, John Wiley, 1973.

${ }^{15}$ Fischbach, H, Breker, H, and Thoenes, G H, Klinische Wochenschrift, 1977, 55, 259.

${ }^{16}$ Gill, D, Clinical Nephrology, 1977, 8, in press

' Mota-Hernández, F, Briseño-Mondragón, E, and Gordillo-Paniagua, G, Nephron, 1976, 16, 272.

${ }^{18}$ Hinglais, N, Garcia-Torres, R, and Kleinknecht, D, American fournal of Medicine, 1974, 56, 52.

19 Baldwin, D S, et al, Annals of Internal Medicine, 1974, 80, 342.

${ }^{20}$ Baldwin, D S, American Fournal of Medicine, 1977, 62, 1.

${ }^{21}$ Schacht, R G, et al, New England fournal of Medicine, 1977, 295, 977.

${ }^{22}$ Rodriguez-Iturbe, B, et al, Clinical Nephrology, 1976, 5, 197.

\section{The gut, genes, and brain of the schizophrenic}

Medicine often advances through chance observations, and not all are made by professional workers. Who is better placed to observe than relatives? Hence there is much to be said for the Schizophrenia Association of Great Britain, essentially a pressure group encouraging research (even if resisting theories of intrafamily dynamics). Many of its members seem impressed by the value of gluten-free and other diets. This they made clear at the annual meeting last month, which was attended by leading nutritionists, immunologists, and psychiatrists. This evidence from the families seems good enough to warrant careful attention-though it has not been adequate to convince the medical establishment.

The nature of schizophrenia, however, presents problems in the methods used. If-as seems likely-the diagnosis covers a mixed group of disorders then double-blind trials will tend to miss specific treatments while highlighting blunderbuss concepts and remedies. One interesting study was reported by Wetterberg from the Karolinska Institute in Stockholm. The method used to isolate "a syndrome" was based on the study of a pedigree of 5000 individuals in 12 generations and including 47 patients. The sector of the family studied gave results consistent with inheritance through a dominant gene with partial $(20 \%)$ penetrance. The Swedish group has compared families with schizophrenia and porphyria (with measures of uroporphyrinogen-I-synthetase acting as a genetic marker). In a porphyric family with 11 generations identified they showed dominant inheritance with a $10 \%$ incidence of the disease and women suffering more often than men. The sex difference was thought perhaps to be due to differences in steroid metabolism, while variations in the chemical environment acted as the trigger for the porphyria.

The concept of looking at illnesses as analogous was stimulating, but there remained the possibility that an enzymatic defect might occur only in one family-patients studied closely are not necessarily typical. Nevertheless, over 2000 illnesses have been identified genetically already, and Wetterberg rightly pointed out that with new techniques such as gas chromatography and mass spectrometry the search for the schizophrenic needle in the haystack was no longer preposterous.

Unfortunately, there are still discrepancies in reported studies of biochemical features such as enzyme levels. For example, monoamine oxidase concentrations in platelets have generally been reported as being low in schizophrenia, but, 